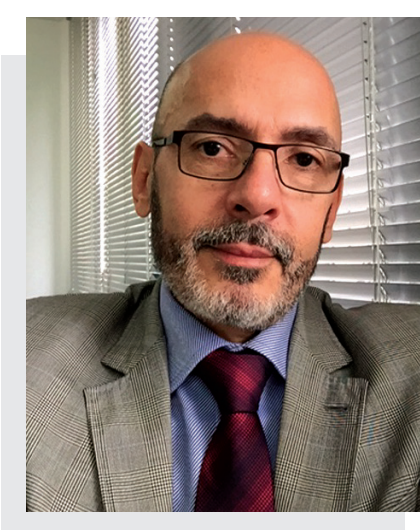

\title{
Obesidade e Fibrilação Atrial
}

\author{
Obesity and Atrial Fibrillation
}

J. Tarcísio Medeiros de Vasconcelos ${ }^{1, *}$

A fibrilação atrial ocupa uma discussão central hoje no universo das arritmias cardíacas. O aumento da expectativa de vida que trouxe um substancial aumento de sua prevalência na população geral ${ }^{1}$ implicou em um aprofundamento marcante na compreensão dos seus mecanismos eletrofisiológicos, na identificação de fatores determinantes ou de potencialização da sua ocorrência e obviamente no desenvolvimento de estratégias efetivas de tratamento e de prevenção das suas complicações. Desde o clássico estudo de Wijffels et al., publicado em 1995 (Atrial Fibrillation Begets Atrial Fibrillation) ${ }^{2}$ demonstrando experimentalmente que frequências atriais artificialmente impostas ao miocárdio atrial implicam em mudanças eletrofisiológicas marcantes determinantes da própria ocorrência da fibrilação atrial, ficou claro que a arritmia uma vez iniciada potencializa a sua própria ocorrência e um processo constante de retroalimentação, cujo desfecho final ao longo do tempo é a instalação da arritmia em uma forma permanente. Contudo para que a fibrilação atrial ocorra é necessária a presença de um ambiente eletrofisiológico adequado, consequente à presença de diversos elementos que agridem o miocárdio atrial sob o aspecto elétrico e estrutural.

A obesidade é um fator identificado como marcador de risco independente para a ocorrência da fibrilação atrial e para sua progressão de uma forma paroxística para permanente. No estudo de Framingham, a obesidade implicou em um risco em torno de 1,5 vez maior para o desenvolvimento de fibrilação atrial; o aumento de 1 ponto no índice de massa corpórea esteve relacionado a um aumento de $4 \%$ na chance de desenvolver arritmia ${ }^{3}$. De modo similar, a obesidade também está implicada na maior chance de evolução da própria arritmia nos indivíduos já portadores de fibrilação atrial. Em um estudo observacional de 21 anos, Tsang et al. demonstraram que os indivíduos obesos com fibrilação atrial paroxística têm 1,5 (IMC 30a 34,9 kg/m²) a 1,9 vez (IMC > 35 kg/m² - obesos mórbidos) mais chances de progressão para fibrilação atrial permanente do que os não obesos ${ }^{4}$. Os mecanismos implicados nesse efeito não são claramente compreendidos. É possível que a liberação de mediadores inflamatórios, fatores neuro-humorais e elementos hemodinâmicos tenham um papel determinante nessa suscetibilidade ${ }^{5-9}$. É possível também que a apneia do sono, frequentemente relacionada à obesidade, possa ter um papel determinante significativo na ocorrência de fibrilação atrial nesses indivíduos ${ }^{10}$.

Por mais que a associação entre obesidade e fibrilação atrial esteja bem reconhecida, os benefícios da perda de peso no tratamento de indivíduos já portadores da arritmia só recentemente começaram a ser avaliados. No estudo LEGACY publicado em $2015^{11}, 355$ indivíduos com índice de massa corpórea $\geq 27 \mathrm{~kg} / \mathrm{m}^{2}$, portadores de fibrilação atrial, foram estimulados a perda ponderal e acompanhados por cerca de 5 anos. A perda de peso igual ou superior a 10\% implicou em uma substancial redução das cargas de fibrilação atrial e em um aumento expressivo da taxa de pacientes livres da arritmia no seguimento. Indivíduos que atingiram essa meta de perda de peso tiveram mais de seis vezes a chance de permanecerem livres de fibrilação atrial do que aqueles que não atingiram esse índice de perda. Em uma publicação mais recente, em complementação ao próprio estudo LEGACY, foi avaliado o impacto da magnitude das perdas ponderais sobre a progressão da forma de exteriorização da fibrilação atrial ${ }^{12}$. Ficou demonstrada uma relação inversa entre a magnitude da perda ponderal e a progressão da arritmia. Pacientes com perda de peso superior ou igual a 10\% tiveram substancialmente uma menor taxa de progressão da fibrilação atrial da forma paroxística para a forma persistente, uma maior taxa de regressão da forma persistente para a paroxística e também uma menor taxa de ocorrência da própria arritmia, quando comparados àqueles pacientes que tiveram perda de peso inferior a esse valor.

1.Centro Avançado de Ritmologia e Eletrofisiologia - São Paulo (SP) - Brasil.

*Autor correspondente: tarr@terra.com.br

ORCID: Vasconcelos JTM (iD) https://orcid.org/0000-0002-5152-2648 
O impacto da obesidade também se faz sentir nos resultados do próprio tratamento da fibrilação atrial. Em pacientes obesos e obesos mórbidos submetidos a tratamento intervencionista mediante ablação percutânea por isolamento das veias pulmonares, as taxas de insucesso têm se mostrado elevadas, atingindo algo em torno de $60 \% \mathrm{em} 1$ ano de seguimento ${ }^{13}$. O tratamento da obesidade previamente ao tratamento da fibrilação atrial por ablação aparenta melhorar os resultados do procedimento. Em um estudo realizado por Donnellan et al., publicado em $2019^{14}, 51$ obesos mórbidos com IMC $\geq 40 \mathrm{~kg} / \mathrm{m}^{2}$, portadores de fibrilação atrial, foram submetidos a tratamento da obesidade por cirurgia bariátrica previamente à realização de ablação percutânea para tratamento da arritmia. A amostra foi pareada em um padrão 2:1 conforme idade e sexo a 102 pacientes não obesos e 102 pacientes obesos mórbidos não tratados por cirurgia bariátrica, também submetidos a ablação percutânea. Em cerca de 30 meses de seguimento, as taxas de recorrência de fibrilação atrial foram de $20 \%$ nos obesos tratados por cirurgia bariátrica, $24 \%$ nos não obesos e $55 \%$ nos obesos mórbidos não tratados por cirurgia bariátrica $(\mathrm{p}<0,0001)$.

Em síntese, a obesidade aumenta o risco para ocorrência de fibrilação atrial, acelera a sua progressão naqueles pacientes já portadores da arritmia e piora os resultados do tratamento. Por sua vez, a redução ponderal em portadores de fibrilação atrial reduz o risco para sua ocorrência em não portadores da arritmia, interfere na história natural naqueles indivíduos portadores da arritmia e melhora os resultados do tratamento por ablação. A redução ponderal agressiva, portanto, deve ser uma pedra fundamental na prevenção e tratamento dessa multifacetada arritmia.

\section{REFERÊNCIAS}

1. Staerk L, Wang B, Preis SR, Larson MG, Lubitz SA, Ellinor, PT. Lifetime risk of atrial fibrillation according to optimal, borderline, or elevated levels of risk factors: cohort study based on longitudinal data from the Framingham Heart Study. BMJ. 2018;361:1-10. https://doi. org/10.1136/bmj.k1453

2. Wijfels MCEF, Kirchhof CJHJ, Dorland R, Allessie MA. Atrial Fibrillation Begets Atrial Fibrillation. A Study in Awake Chronically Instrumented Goats. Circulation. 1995;92(7):1954-68. https://doi.org/10.1161/01.cir.92.7.1954

3. Wang TJ, Parise H, Levy D, D'Agostino RB, WolfPA, Vasan RS, etal. Obesity and the risk of new-onset atrial fibrillation.JAMA. 2004;292(20):24717. https://doi.org/10.1001/jama.292.20.2471

4. Tsang TS, Barnes ME, Miyasaka Y, Cha SS, Bailey KR, Verzosa GC, et al. Obesity as a risk factor for the progression of paroxysmal to permanent atrial fibrillation: a longitudinal cohort study of 21 years. Eur HeartJ. 2008;29(18):2227-33. https://doi.org/10.1093/eurheartj/ehn324

5. Juge-Aubry CE, Henrichot E, Meier CA. Adipose tissue: a regulator of inflammation. Best Pract Res Clin Endocrinol Metab. 2005;19(4):54766. https://doi.org/10.1016/j.beem.2005.07.009

6. Warnberg J, Nova E, Moreno LA, Romeo J, Mesana MI, Ruiz JR, et al. Inflammatory proteins are related to total and abdominal adiposity in a healthy adolescent population: the AVENA Study. Am J Clin Nutr. 2006;84(3):505-12. https://doi.org/10.1093/ajcn/84.3.505

7. Kahn SE, Zinman B, Haffner SM, O'Neill MC, Kravitz BG, Yu D, et al. Obesity is a major determinant of the association of C-reactive protein levels and the metabolic syndrome in type 2 diabetes. Diabetes. 2006;55(8):2357-64. https://doi.org/10.2337/db06-0116

8. Wang TJ, Larson MG, Levy D, Benjamin EJ, Leip EP, Wilson PW, et al. Impact of obesity on plasma natriuretic peptide levels. Circulation. 2004;109(5):594-600. https://doi.org/10.1161/01.CIR.0000112582.16683.EA

9. Wildman RP, Farhat GN, Patel AS, Mackey RH, Brockwell S, Thompson T, et al. Weight change is associated with change in arterial stiffness among healthy young adults. Hypertension. 2005;45(2):187-92. https://doi.org/10.1161/01.HYP.0000152200.10578.5d

10. Gami AS, Hodge DO, Herges RM, Olson EJ, Nykodym J, Kara T, et al. Obstructive sleep apnea, obesity, and the risk of incident atrial fibrillation. J Am Coll Cardiol. 2007;49(5):565-71. https://doi.org/10.1016/j.jacc.2006.08.060

11. Pathak RK, Middeldorp ME, Meredith M, Mehta AB, Mahajan R, Wong CX, et al. Long-term effect of goal directed weight management in an atrial fibrillation cohort: a long-term follow-up study (LEGACY). J Am Coll Cardiol. 2015;65(20):2159-69. https://doi.org/10.1016/j. jacc.2015.03.002

12. Middeldorp ME, Pathak RK, Meredith M, Mehta AB, ElliottAD, Mahajan R, et al. Prevention and regressive effect of weight-loss and risk factor modification on atrial fibrillation: the REVERSE-AF study. Europace. 2018;20(12):1929-35. https://doi.org/10.1093/europace/euy117

13. Sivasambu B, Balouch MA, Zghaib T, Bajwa RJ, Chrispin J, Berger RD, et al. Increased rates of atrial fibrillation recurrence following pulmonary vein isolation in overweight and obese patients. J Cardiovasc Electrophysiol. 2018;29(2):239-45. https://doi.org/10.1111/jce.13388

14. Donnellan E, Wazni O, Kanj M, Hussein A, Baranowski B, Lindsay B, et al. Outcomes of atrial fibrillation ablation in morbidly obese patients following bariatric surgery compared with a nonobese cohort. Circ Arrhythm Electrophysiol. 2019;12(10):1-6. https://doi.org/10.1161/ CIRCEP.119.007598 